\title{
COVID 19; A SURGICAL PERSPECTIVE
}

\author{
Anwar UI Haque
}

The novel corona virus disease started at Wuhan in Hubei province of China as pneumonia of unknown origin and it was found that the causative organism of Coronavirus Disease 2019(COVID-19) was Severe Acute Respiratory Syndrome Coronavirus type 2 (SARS-CoV-2). Healthcare systems all over the world nearly collapsed due to unprecedented spread of the virus and the disease COVID-19 was notified as a pandemic by WHO on March 11,2020., Until October 2020 more than 37 million cases and more than one million deaths have been reported worldwide from this highly contagious disease.

Along with global socioeconomic implications and impact on various sectors of life. This novel corona virus disease had great impact on practice of surgical and allied disciplines widely ranging from staff placement and workplace issues ,prioritization of procedures, transfer of virus during surgeries, to surgical training and education.

Surgeons and related staff along with operating on emergency surgical patients were also given duties to take care of COVID patients throughout hospitals and had to undertake trainings on critical care. Health staff, essential resources and beds of healthcare facilities had to be rearranged and allocated to the critical management of patients suffering from COVID-19. This lead to stoppage of almost all non emergency, elective procedures. A significant backlog thus created lead to unrest and anxiety among patients. Herod P.J.J. noted that $30 \%$ of patients, operations of whom were cancelled due to COVID-19 disease pressures during winter suffered from extreme stress and frustration while $59 \%$ reported moderate or severe concern about an aggravation of their condition ${ }^{3}$.

Undergraduate and postgraduate surgical training was disrupted due to suspension of normal training rotations and cancellation of many surgical conferences. Smart learning technologies including online classes, virtual rounds ,clinical case discussions and live webinars helped in learning to some extent.

Correspondence: Professor Dr. Anwar Ul Haque

Professor Incharge Surgical B Unit Saidu Medical College I Saidu Teaching Hospital Swat.

Received: December12th, 2019 , Accepted: January 12, 2020
Laboratory and clinical research has to suspended. Apart from other protective measures changes had to be made to ventilation system of operation theaters. While performing long operations in full PPEs was exhausting, lack of sleep and anxiety also lead to slower cognitive processing and decision making and thus increasing risk of error $^{4}$. Such a demanding environment also lead to burn out amongst clinicians.

As the number of cases declined, elective surgical procedures have to be started because these cannot be postponded indefinitely to prevent anxiety and stress to patients and further deterioration of their disease as well as deleterious effects on livelihood of surgical health care professionals. Different countries have different approach to timing and protocols for starting elective procedures and what necessary arrangements be made for protection of patients and safety of staff of healthcare facilities when routine work starts. It is of great importance to develop evidence based protocols for testing , surgical/procedural risk mitigation, organizing flow of patients and contamination control to keep surgical practice safe.

The role of acquired immunity after infection in protection and its duration of protection is not clear. Carriers of virus who are asymptomatic may be a source of spread for the time until a vaccine is available. So for the time being our objectives are to;

Screen all patients by testing to ensure that who could have safe surgery

Protect COVID negative patients who are undergoing elective surgeries

Prevent health care workers from getting corona virus.

Important principles of medical ethics as narrated by Beauchamp and Childress ${ }^{5}$ including Beneficence, Nonmaleficence, Autonomy and Justice should be followed by surgeons for ethical practice and surgeons should deal patients fairly and honesty with compassion and respect. While taking care of an individual patient we should take into account all the available resources and needs of all the patients to maintain Social justice in Healthcare, Altruism has been demonstrated by surgeons which is the selfless concern for the wellbeing of others. 
Surgeons and related staff were redistributed to assist other frontline services in critical care and emergency departments and they selflessly placed themselves at risk to serve patients and help their colleagues. They shared surgical experience and worked hard to provide nonsurgical care competently by improving their skills and support from other colleagues. They have safely operated and take care of SARS-Cov-2positive patients, while maintaining nosocomial transmission to healthcare workers to minimum level ${ }^{6,7.8}$ At the same time infection prevention and control practices should be robust, patients risk assessed by COVID-19 testing and health staff kept safe with personel- protective equipment(PPE) and environmental shielding to prevent depletion of surgical care.

An important issue was to when and how to start elective lanesthetic procedures and the type organizational changes to be made for patient safety and protection of health staff when clinics and hospital OPDs start functioning. Although the wave has flattened out in most countries, still a large number of cases are being reported from various parts of world and in Pakistan the incidence is again rising. To keep elective surgeries safe, Kaye etal ${ }^{9}$ have defined six subsets for the creation of guideline proposals:

- Surgical risk management and risk stratifying

- Perioperative and anaesthesia management

- $\quad$ Preoperative testing and screening

- Perioperative pharmacologic prophylaxis

- Clinical management and contamination control

- Patient information and patient consent

During surgery of COVID -19-positive patient, increasing level of surgical complexity, use of general anesthesia and longer duration of surgery correlate with a poorer clinical outcome ${ }^{10,11}$. So it is important to make sure to the maximum limit that a patient undergoing an elective operation is COVID negative .Projections for transmission dynamics of COVID-19 show that recurrent post pandemic outbreaks are to be expected until herd immunity is acquired, which may take until $2024 .{ }^{12}$

As long as vaccine is not available protective measures should be followed. Masks, hand disinfection, temperature checking, social distancing and spacing between consultations should be routine at offices and clinics. History should cover symptoms of COVID-19, travelling, exposure to infected patients in last 14 days, comorbidities, etc. RT-PCR, Complete blood picture to check for COVID-19 related changes (like leukocpenia and lymphopenia ), coagulation tests, renal function tests, C Reactive Protien, blood sugar and chest X-ray should be part of standard preoperative investigations ${ }^{13}$. Infection prevention protocols including PPEs ,surface cleaning protocols, negative pressure systems should be strictly followed in operation theaters. Regional /tumescent anaesthesia is better to avoid invasion of tracheal-bronchial tract. An active scavenging system should be there for exhalation gases and circuits should have two HME filters. Patients should be given information regarding COVID-19 and its associated risks in the perioperative period and informed consent taken.

Any patient who develop COVID-19 compatible symptoms needs to be immediately referred to COVID-19 team for further care.

All health care facilities including air conditioners should be properly prepared and disinfected. Duct based air conditioners that distribute and share air between different clinical sections should not be used without adequate decontamination( UVC, ionization, HEPA filters, etc ) and keeping in view future outbreaks of the disease changes should be made in operation theaters like negative pressure ventilation, split air conditioning units etc,

All health care workers have emerged more united from the pandemic and will work with same zeal in future. Different elective surgical specialities should be better prepared for possible second wave of the pandemic.

\section{Refrences;}

1. Zhu N, Zhang D, Wang W, Li X, Yang B, Song J, et al. A novel coronavirus from patients with pneumonia in China, 2019. N Engl J Med. 2020;382:727-733

2. Sohrabi C, Alsafi Z, O'Neill N, Khan M, Kerwan A, AlJabir A et al. World Health Organization declares global emergency: A review of the 2019 novel coronavirus (COVID-19) Int J Surg Lond. Engl. 2020;76:71-76

3. Herrod PJJ, Adiamah A, Boyd-Carson H, Daliya P, ElSharkawy AM, Sarmah PB et al. Winter cancellations of elective surgical procedures in the UK: a questionnaire survey of patients on the economic and psychological impact. BMJ Open. 2019;9:21-28 
4. Parry DA, Oeppen RS, Amin MSA. Sleep: its importance and the effects of deprivation on surgeons and other healthcare professionals. $\mathrm{Br} \mathrm{J}$ Oral Maxillofac Surg. 2018;56:663-666

5. Beauchamp T, Childress J. Principles of biomedical ethics: marking its fortieth anniversary. Am J Bioeth.2019;19 (11):.9-12.

6. Prachand VN, Milner R, Angelos P, Posner MC, Fung JJ, Agrawal N, et al. Medically necessary, timesensitive procedures: scoring system to ethically and efficiently manage resource scarcity and provider risk during the COVID-19 pandemic. J Am Coll Surg.2020;231:281-88.

7. Al-Jabir A, Kerwan A, Nicola M, Alsafi Z, Khan M, Sohrabi C,et al. Impact of the Coronavirus (COVID19) pandemic on surgical practice - Part 1(review article). Int J Surg. 2020, Jul; 79: 168-179

8. Al-Jabir A, Kerwan A, Nicola M, Alsafi Z, Khan M, Sohrabi C,et al. Impact of the Coronavirus (COVID19) pandemic on surgical practice - Part 2 (surgical prioritisation). Int. J Surg. 79 . https://doi.org/10.1016/j.ijsu.2020.05.002

9. Kaye K, Paprottka F, Escudero R, Casabona G, Montes J, Fakin R,et al. Elective, Non-urgent Procedures and Aesthetic Surgery in the Wake of SARSCOVID-19: Considerations Regarding Safety, Feasibility and Impact on Clinical Management. Aesthetic Plast Surg. 2020;14: 129-132.
10. Aminian A, Safari S, Jahromi RA, Ghorbani M, Delaney CP. COVID-19 outbreak and surgical practice: unexpected fatality in perioperative period. An $\mathrm{n}$ Surg. ht t ps:// d o i.org/10. 1101/2020.04.05.2005:43-61

11. Lei S, Jiang F, Su W, Chen C, Chen J, Mei W et al. Clinical characteristics and outcomes of patients undergoing surgeries during the incubation period of COVID-19 infection. EClinicalMedicine, 21 (2020), 1003-31

12. Xiao AT, Tong $Y X$, Zhang S . False-negative of RT$\mathrm{PCR}$ and prolonged nucleic acid conversion in COVID-19: rather than recurrence. J Med Virol. https://doi.org/10.1002/jmv.258-63

13. Rajan N, Joshi GP. The Covid-19: role of ambulatory surgery facilities in this global pandemic. Anesth A $\mathrm{n}$ a I g https://doi.org/10.1213/ane.0000000000004847

14. Khan W. Corona Virus Disease 2019 .J Saidu Med Coll Swat 2020;10(1):01-02. 